\title{
Exploring the Role of Mental Imagery in the Experience of Self-injury: An Interpretative Phenomenological Analysis
}

P Dargan, G Reid, S Hodge

North Staffordshire Combined Healthcare NHS Trust, Harplands Hospital, Hilton Road, Harpfields, Stoke-on-Trent, Staffordshire, ST4 6TH, Dr Peter Dargan, Clinical Psychologist

Lancashire Care NHS Foundation Trust, Scarisbrick Centre, Ormskirk and District Hospital, Wigan Road, Ormskirk, L39 2AZ, Dr Graeme Reid, Clinical Psychologist

Doctorate in Clinical Psychology, Division of Health Research, Furness College, Lancaster University, Lancaster, LA1 4YG, Dr Suzanne Hodge, Lecturer in Health Research

Correspondence to: Peter.Dargan2@northstaffs.nhs.uk

The lead author affirms that the manuscript is an honest, accurate and transparent account of the study being reported, and that no important aspects of the study have been omitted.

All authors have completed the ICMJE uniform disclosure form at www.icmje.org/coi_disclosure.pdf and declare: no support from any organisation for the submitted work; no financial relationships with any organisations that might have an interest in the submitted work in the previous three years; no other relationships or activities that could appear to have influenced the submitted work. 


\begin{abstract}
Aims: Research has implicated causal, mediating and meaningful roles for mental imagery in the experience of self-injury. The present study aimed to further the understanding of this relationship through exploring the lived experiences of mental imagery from the perspective of those who self-injure. Methods: This study employed an inductive qualitative design using semi-structured interviews and Interpretative Phenomenological Analysis (IPA). Participants: Ten participants were recruited from universities in North West England. Results: Three main themes were identified from the analysis: (i) The origins and precipitants of self-injurious imagery; (ii) What it is like to experience self-injurious imagery; and (iii) The meaning and interpretation of self-injurious imagery. Conclusions: The study findings indicate that mental imagery is an important experience for those who self-injure. Clinical and research implications of the findings are discussed.
\end{abstract}

Keywords: Imagery; self-injury; cognition; IPA 


\section{Mental Imagery}

The ability to experience cognitive or 'mental' imagery is a key aspect of human consciousness. Imagery that is experienced cognitively, in vivo, rather than through external reality, has been defined as "mental representations of something, not by direct perception but by memory or imagination" (Hackmann \& Holmes, 2004, p. 390). And whilst cognitive imagery is predominantly visual (Rice \& Rubin, 2009), it can take place within any sensory modality (Kosslyn, Ganis, \& Thompson, 2001).

Psychological research has consistently implicated cognitive imagery as important within experiences of psychological distress, including: Post-Traumatic Stress Disorder (PTSD) (Ehlers, Hackmann, \& Michael, 2004); Social Phobia (Hackmann, Clark, \& McManus, 2000); Panic Disorder (Ottaviani \& Beck, 1987); Obsessive Compulsive Disorder (OCD) (Speckens, Hackmann, Ehlers, \& Cuthbert, 2007); Depression (Holmes, Crane, Fennell, \& Williams, 2007); Body Dysmorphia (Osman, Cooper, Hackmann, \& Veale, 2004); Eating Disorders (Somerville \& Cooper, 2007); Psychosis (Morrison, 2004); Bipolar Disorder (Holmes, Geddes, Colom, \& Goodwin, 2008) and Addiction (May, Andrade, Panabokke, \& Kavanagh, 2004).

Research regarding imagery and psychological distress has also revealed how attending to the cognitive qualities of mental imagery can provide insights into imagery meaning and function. For example, the vantage point within an image (Nigro \& Neisser, 1983); the sensory modalities involved (Kosslyn et al., 2001); whether an image is deliberately evoked or externally cued (Ehlers \& Clark, 2000); how imagery is cognitively appraised and responded to (Hackmann \& Holmes, 2004); whether imagery is experienced during the day or night (Freeman \& White, 2002); and whether imagery is literal or abstract 
and metaphorical (Hackmann, Bennett-Levy, \& Holmes, 2011) have all been found to affect how cognitive imagery is experienced.

\section{Imagery and self-injury}

A current and developing area of research is the relationship between cognitive imagery and self-injury. Self-injury can be defined as "the intentional destruction of body tissue without suicidal intent and for purposes not socially sanctioned" (Klonsky \& Muehlenkamp, 2007, p.1045). Self-injury may have a strong relationship with cognitive imagery in that it typically involves creating visual changes to the body, and encompasses a diverse range of sensory sensations (Groschwitz \& Plener, 2012). For instance, the sight and imagery of blood has been described as of prime importance for at least $50 \%$ of those who self-injure (Glenn \& Klonsky, 2010), as it can often be an indicator that a cut was done 'correctly' (Himber, 1994), or can lead to feelings of relief and calmness (Van Moffaert, 1990).

Whilst research has yet to directly explore whether those who self-injure experience cognitive imagery related to these personally meaningful phenomena, studies have observed and commented upon this relationship (e.g. Harris, 2000), and cognitive imagery has been noted to be important within first person accounts of self-injury, such as through containing personal meaning (Spandler, 1996) or causing psychological distress (Leatham, 2006). Neuroimaging studies also suggest that cognitive imagery has an important role in self-injury, in that self-injurious-related imagery mediates altered psychophysiological arousal in those who self-injure when compared to control groups (e.g. Kraus et al., 2010).

Despite research suggesting that for some people self-injurious mental imagery exists and has clear psychophysiological implications, as yet no study has sought to explore how 
people who identify themselves as self-injurers experience imagery; what the role of this imagery might be; and what it means to those experiencing it.

\section{Aims of the research:}

This study aimed to provide further insight regarding the presence, impact, and implications of self-injurious mental imagery. Additionally, the research aimed to produce an ecologically valid sense of how such imagery is incorporated into lived experience. Finally, the study aimed to bring the views of those who self-injure to the forefront of the evidence base.

\section{Method}

\section{Design}

A qualitative Interpretative Phenomenological Analysis (IPA) design was employed utilising semi-structured interviews. IPA was deemed the most appropriate approach due to its focus upon how people make sense of major life experiences through the process of interpretation, and because it has commonly been used to explore issues of psychological distress, life transitions and identity (Smith, Flowers, \& Larkin, 2009). The interview schedule was developed in consultation with an advisory group who had personal experience of self-injury $(\mathrm{N}=8)$, recruited through an online self-harm support website.

\section{Context and Researchers}

The research took place as part of a larger research project, contributing toward the completion of a Doctorate in Clinical Psychology for the first author, who was the principal investigator. The second and third authors provided supervision for the research in addition to 
checks for adherence and competence regarding the principles of the research methodology employed. Whilst the research team share a belief in the use of psychological theory and research in the understanding of human behaviour and psychological distress, there were no preconceptions regarding the roles (if any) of mental imagery in the experience of selfinjurious behaviour.

\section{Participants}

The study sample was comprised of working-age adults who had personal experience of self-injury. It has been argued that those who self-injure do not consistently present to healthcare services (MaSH, 2012), and are a diagnostically heterogeneous group (Klonsky \& Muehlenkamp, 2007), therefore participants were recruited from the general population. University students were identified as a suitable population for the research as these individuals have been identified as at much lower risk of harm, hospitalisation and suicide than other self-injury populations (Mahadevan, Hawton, \& Casey, 2010). Three universities in the North-West of England consented to advertise the research. A final sample of ten participants took part in the study; seven females and three males. All provided written informed consent and were invited to select pseudonyms for their anonymous representation in the research findings. Links with University counselling services were made for any participants who experienced distress from taking part in the research. Table 1 displays participant characteristics.

Insert Table 1 here

\section{Ethical Approval}


Ethical approval for the study was provided by Lancaster University Faculty of Health and Medicine Research Ethics Committee (ref: FHMREC1100060).

\section{Data Collection \& Analysis}

Standard analytic processes for IPA were followed (Smith et al., 2009). Interviews were conducted, transcribed and analysed by the first author. The researcher analysed each transcript in turn, making initial notations beside each transcript which recorded his immediate reflections and observations. From these initial notations emergent themes were identified within each transcript. Major themes were then developed for each participant by joining together semantically related emergent themes. A summary was written for each major theme, thus providing an overall narrative of experience for each participant. Once this process had been completed for each transcript, each major theme was juxtaposed with others within and across participant accounts, and connected to those that carried similarities in content or meaning. These interrelated themes were thus worked into a set of super-ordinate themes representing the entire sample. Super-ordinate themes were labelled with titles that reflected both participants' experiences and the researcher's interpretation. The quality and coherence of the final themes were appraised by the second and third authors in order to strengthen the validity of the analytical process.

\section{Findings}

All participants identified with the concept of imagery, describing varying experiences. Three super-ordinate themes were identified from the analysis.

An additional theme 'The biographical context of self-injury' was also identified within the research. Whilst this theme was not deemed to be directly relevant to the research question, it was important in contextualising participants' narratives of self-injury and 
imagery, and understanding how individual accounts were related to others within the analysis.

This additional theme revealed that participants' self-injury typically emerged following traumatic life experiences; themes of chronic illness, bereavement and loss, childhood and adult sexual abuse, mental health difficulties, significant life events and change were common. For others self-injury represented a developmental stage that reemerged following trauma and extreme stress in adult life. All participants described first engaging in self-injury during childhood or teenage years, but stressed that it evolved in line with their life circumstances, settling down for periods and re-emerging when needed, or changing in function (e.g. from a "coping mechanism" to a way of experiencing pleasurable sensations).

\section{Theme one: The origins and precipitants of self-injurious imagery}

This initial theme captures participants' understandings of how imagery related to self-injury had originated in their lives. Several participants had begun to experience imagery almost immediately after they started to self-injure:

I'd say sort of after the first few times you do it [...] obviously if you've never had experience of self-harm you don't see the images because you don't know what it's like. (Cara)

For others the relationship between the emergence of self-injury and the experience of imagery felt more complex. At least three participants felt that they had only begun experiencing imagery related to self-injury after their self-injury had become more intense or severe: 
I don't...can't remember any imagery from when I was harming around the age of sixteen but I was barely doing anything. (Fry)

A key finding regarding the onset and experience of mental imagery was that it could be both deliberately evoked and engaged with, or "triggered" into consciousness by internal or external factors. It appeared that whilst all participants could experience the onset of imagery both purposively or spontaneously, the degree of choice in experiencing imagery could affect whether it was experienced as pleasant or intrusive. Michelle's experiences reflect this dichotomy. First she describes "a pleasant daydream" of having an accident and hurting herself:

Say if I'm tired and I don't want to go to work I imagine slipping and falling [...] it's a fantasy of being incapacitated really. (Michelle)

She then contrasts this with experiencing involuntary or "triggered" self-injurious imagery:

It's extremely overwhelming [...] like a really intense situation where you don't have any skills available to combat it $[. .$.$] and that's when it comes it's like I'm fighting$ with myself [...] which I guess is more self-injury [imagery] but it just so physically blocks me up with like that much anger and it's all self-directed anger so...and the accident type stuff is much very calm it's almost [...] like I'm musing over like what it would be like to fall and break my leg. (Michelle)

The relationship between whether imagery was deliberately evoked or cued and whether it was experienced as pleasant or unpleasant did not appear to be entirely mutually exclusive within participants' narratives. Participants explained that even pleasant imagery that was spontaneously triggered could lead to mixed feelings, dissonance or harming themselves: 
If it [positive imagery] comes into my head it does stick more....like I said it could be one day it happens and then it'll be in the back of my mind like it's just there and [...] I keep...that image never fully goes away and I keep...imagining that release I would get from it $[\ldots]$ eventually after a few days it'll lead to self-harm. (Alan)

The rationale for deliberately evoking imagery often included wanting to mirror the ritualistic quality of self-injury, and experiencing physical arousal. Jenny described how for her engaging in imagery fulfilled some of the previous functions of self-injury:

It's a calming routine thing for me now so I'm used to kind of going to bed, think about the sensation for a while and that erm maybe raises some sort of...it's sort of like doing a bit of intense exercise and then it tiring you out $[\ldots]$ and then you go straight to sleep after. (Jenny)

The range of conditions described for intrusive imagery were by comparison much more varied across participants, and commonly included elements such as a significant change in mood (particularly depression); viewing objects or tools commonly associated with self-injury (e.g. razor blades, scissors); the environment and contextual situations (e.g. nightclubs, social events); or references to self-harm in the news or wider media. It appeared for each participant that personal cues or triggers existed for their cognitive imagery.

An important interpretation emerging from participant accounts is the complex relationship between feeling that imagery had been externally or internally triggered, and that urges to self-injure had then been triggered by the experience of imagery. The experience of imagery seemed for several participants to be the mediator between a trigger in the environment and the outcome of self-injury, whereas others felt that experiencing imagery rarely or never resulted in self-injury, or urges to self-injure. This included both participants 
who were no longer actively self-injuring at the time of interview and those who felt it was still prominent in their lives:

It's not like stimulus response type thing so like if erm I randomly imagine the shouty noise [image] that wouldn't make me self-injure if you see what I mean [...] the only main stimulus I have is overwhelming it's like a feeling of overwhelmment. (Sally)

Participants who did feel that imagery could become a trigger or precipitant of an urge to injure themselves were keen to point out that this link was far from a straightforward or reactive process. As Alan explained, his imagery could linger for several days, sometimes resulting in self-injury and sometimes not:

it might be like the Tuesday it's in my head and then it sticks there and then I might have a dream about it and by Thursday I might do it. (Alan)

\section{Theme two: What it is like to experience self-injurious imagery}

This theme encapsulates the content of participants' self-injury related imagery, and what it was like for them to experience it. All of the participants described experiencing cognitive imagery related to self-injury, and this appeared to be a highly personal and individual experience:

One image is one where I've got streaks of blood dripping down from my finger tips after I flicked it erm....and every direction I can actually recall the number of cuts there were at that point in time and where each of them are. (Wendy) 
Many participants reported that imagery was not just confined to direct examples of self-injury but also included things that felt tangentially related. For example some participants described experiencing images of accidents:

if I'm on a hike for instance I'll imagine falling off a bridge and breaking my leg (Michelle).

or images of suicide or not existing:

the idea of like slitting my throat and just bleeding out.

The viewing perspectives participants experienced seemed to be particularly important. Participants described experiencing imagery from both a first person and a third person perspective. A trend across all narratives was that images from a first person perspective were often derived from actual experiences, particularly memories of past instances of self-injury. In contrast images that involved the third person perspective did not appear to be derived from participants' direct experiences and instead portrayed a novel situation. Both John and Kirsty noticed this contrast within their experiences. John felt that this resulted from a difference in lived experience, whereas Kirsty felt that her third person imagery was much less desired and based on the evaluation of others, which accounted for differences in perspective.

The traffic one it's third person and with hurting it's first [...] I've done the hurting but I've never been hit by a car so I suppose that one is much more hypothetical. (John)

The reason I go to that image especially with it being third person is that one of the hardest things for me is to think that other people see me as weak or other people see me as vulnerable so it's quite a tormenting image. (Kirsty) 
The experience of imagery also encompassed a range of sensory modalities. For nine of the participants imagery was described as primarily visual, but also included physical or tactile sensations, whereas for one participant it was more auditory and kinaesthetic. Some participants highlighted how imagery that was primarily visual could also evoke powerful physical sensations:

I was doing something to my leg and I remember just lying there afterward and it felt like I was being kind of electrified down my leg and it was kind of a burning lightening sensation and that's erm an image I return to quite often [...] I never got smells or tastes or sounds or anything...but I definitely imagine the sight quite vividly and also the feeling of what it would be like. (Jenny)

These pleasant sensations were often localised to the area of the body where participants would usually cut or harm themselves, however pleasant imagery could also leave them feeling frustrated, because these sensations were not enough to satisfy urges to self-injure.

For all participants the experience of imagery was complex and multifaceted, and gave rise to mixed feelings and responses. For example, evoking both pleasant sensations and frustration, or imagining the desired effects of self-injury yet feeling guilty or regretful. Often this could reflect participants' own personal dilemmas in their relationship with selfinjury.

\section{Theme three: The meaning and interpretation of imagery}

As imagery could be a frequent and personally meaningful experience, and one which participants recognised as being out of the ordinary, all of those involved in the research 
reflected upon its novel nature, what they felt the role of their imagery was, and their interpretations of these experiences.

Several participants described feeling there were links between their imagery and personal beliefs, particularly how they viewed and felt about themselves:

Thoughts of worthlessness about myself if you see what I mean because [imagery] was symbolic of me being pretty worthless [...] so I suppose it ties in with those thoughts I have about myself. (Sally)

Many participants also stated that their images possessed an element of "fantasy" whereby certain aspects of the image would be distorted in reflection of personal preferences or their relationship with self-injury:

When I think about it there's never as much blood as there is in real life... which kind of adds to the fantasy element because it gets in the way when it's bleeding [...] I don't imagine burns a lot just if I do them they're very they're very like...what's the word sort of standard shapes rather than what burns actually are [...] round messy things. (Jenny)

Some participants felt that the meanings contained within their imagery were symbolic. Sally felt that the imagery she experienced had stemmed from some of the most challenging experiences in her life, and may have been personifications of the worst elements. During the interview she attempted to understand why one of her images appeared to embody the voice of an abusive ex partner:

this is the weird thing he well he wasn't there he wasn't with me it wasn't something that I did in front of him" (Sally)

She wondered about the symbolism of these images: 
It's like a chastisement of myself [...] actually invoking that person that sound that negativity [...] or it might be like a motivator I don't know [...] because it's almost reminding me of that ultimate abject feeling of complete horribleness [...] maybe it's a way of getting me to go through with what I've sat down to do. (Sally)

Many participants described experiencing images that they interpreted as containing negative self-appraisals. Several people stated that negative imagery often encapsulated sociocultural motifs of what it means to be a self-harmer, and fears of how they might be perceived:

they contain all the qualities I would like people to least see in me [...] a whingeing whining attention-seeking person who's always miserable (John)

The impact of self-injury upon loved ones also appeared to be a particular focus of imagery:

he looked terrible I remember the expression on his face and things like that (Wendy)

However, whilst this form of negative imagery could mean participants felt more averse to wanting to self-injure, several participants reported that negative images were more transient than images representing the positive aspects of self-injury:

Whereas that image is starting to fade a little bit and I guess it has held me back [from self-injuring] I don't think it will in the future because it's not got the same feeling as the memories of self-harm. As much as I do feel terrible about it it's not as intense. (Alan)

These mixed feelings may reflect how participants tried to make sense of imagery. Several participants expressed worries about experiencing imagery. Despite being accepting of their self-injury this did not always translate into their feelings about personal imagery. 
Participants described how imagery could represent a taboo and that it was rarely discussed by those who self-injure, and/or expressed fears that it might lead others to self-harm.

\section{Discussion}

Many of the findings of this study reflect and further inform previous research. A particularly clear finding was that deliberately evoked imagery tended to be experienced as pleasant and less likely to result in self-injury, whereas imagery that was externally cued was more likely to be experienced as unpleasant and could lead to less controllable urges to selfinjure. Images which were perceived to be 'triggered' or intrusive appeared to determine the experience of psychological distress, rather than the content of the image (i.e. images could be 'positive' but still feel unwanted or distressing due to their spontaneous nature). This is consistent with previous cognitive research indicating that cued imagery has a tendency to be driven by anxiety or threat (Ehlers \& Clark, 2000). These findings may further inform the findings of neuroimaging studies (e.g. Kraus et al., 2010) in that participants described both increased and decreased psychophysiological arousal in relation to imagery, however the present findings suggest that both the personal significance and perceived intrusiveness of mental imagery mediates arousal above image content.

The perspective participants experienced in their imagery also appeared to be of significance. Whilst cognitive images could span both first and third person perspectives, all participant images that involved actively self-injuring were from the first person perspective. Third person perspective images tended to represent events which had never occurred (e.g. accidents), or encompassed undesired qualities (e.g. negative self-perceptions). This supports the findings of previous cognitive research indicating that the observer perspective tends to occur in unwanted or distressing imagery (e.g. Hackmann, 2000), and that images from the 
field perspective are rooted in autobiographical memory and lived experience (Nigro \& Neisser, 1983). However, as described by Rice and Rubin (2009) these findings should be interpreted with caution as some participants described how their images could shift perspective, and were not consistently fixed. Cognitive research regarding the link between imagery and autobiographical memory (Hackmann \& Holmes, 2004) may also explain why certain participants did not experience imagery until they felt self-injury had reached a certain level of intensity or significance, as first person images most often stem from personally meaningful life experiences.

Two particularly novel findings to emerge from the research concerned the fantasy elements of self-injury imagery, and the taboo of imagery within the context of self-injury. Nearly all of the participants reported that certain qualities of the imagery they experienced reflected personal preferences and desires that were not present in reality, and this was perhaps most striking when participants experienced imagery based upon memories or contrasted their images to reality. Participants noted qualities in their imagery such as: a complete absence of blood, or much more blood than in reality; cuts and burns being "neat" or "uniform" shapes whereas in reality they are "messy"; seeing parts of their bodies without cuts or scars as if they were "cutting for the first time"; or imagining inflicting levels of damage that would be risky or undesirable in real life. These nuanced distortions reveal a depth of personal significance related to self-injury that may not be evident from purely descriptive accounts of the behaviour

\section{Clinical Implications}

The current study may have several implications for therapists and mental health services who offer assessment and interventions to those who self-injure. The broadest conclusion that can be drawn is that cognitive images are both experienced by and 
meaningful to at least a sub-set of those who self-injure. Consequently raising awareness of these experiences and ensuring they are asked about by clinicians throughout assessment, formulation and intervention may be important. Exploring imagery with participants could also potentially aid understanding of meanings and beliefs regarding self-injury. However, participants who commented on the taboo nature of self-injurious cognitive imagery also reported that discussing these issues could be a challenge. Therefore the findings suggest that finding/discussing safe and appropriate ways to have conversations around cognitive imagery with those who self-injure may be particularly important, and that clients may experience a sense of aversion or shame in regards to discussing their imagery experiences.

Of more specific relevance to cognitive therapy is the fact that these findings reinforce those of previous cognitive imagery research. This emerging evidence base suggests there may be a role for cognitive imagery techniques in interventions for distressing selfinjurious behaviour. This would be a useful focus of further research.

\section{Methodological considerations}

Whilst the study achieved its aims of further understanding self-injurious related imagery, it is recognised that those who took part in the research may have done so because of their affinity with mental imagery, and it may not be a phenomenon consistent across the self-injury experience. Future research can help to clarify this.

The study had a number of methodological strengths; the ecological relevance of the findings in contextualising imagery within lived experience, the involvement of expert consultants in the research design, and the transparency across data collection and analysis. 


\section{Conclusions}

This study has explored the experience of mental imagery related to self-injury from the perspectives of those with personal experience of the phenomenon. The findings support previous imagery research, suggesting that phenomenological qualities such as image perspective, psychophysiological arousal, and the role of external cues can affect the interpretation and experience of imagery. The study has also produced two particularly novel findings: that self-injury imagery encompasses elements of 'fantasy' that reflect important personal meanings, and that imagery is experienced as taboo within the context of self-injury. Both findings may present challenges and opportunities for clinical service provision and for future research. 


\section{References}

Ehlers, A., \& Clark, D. M. (2000). A cognitive model of posttraumatic stress disorder. [Research Support, Non-U.S. Gov't

Review]. Behaviour research and therapy, 38(4), 319-345.

Ehlers, A., Hackmann, A., \& Michael, T. (2004). Intrusive re-experiencing in post-traumatic stress disorder: phenomenology, theory, and therapy. [Research Support, Non-U.S. Gov't

Review]. Memory, 12(4), 403-415. doi: 10.1080/09658210444000025

Freeman, A., \& White, B. (2002). Dreams and the dream image: Using dreams in cognitive therapy. Journal of Cognitive Psychotherapy: An International Quarterly, 16, 39-53.

Glenn, C. R., \& Klonsky, E. D. (2010). The role of seeing blood in non-suicidal self-injury. $J$ Clin Psychol, 66(4), 466-473. doi: 10.1002/jclp.20661

Groschwitz, R. C., \& Plener, P. L. (2012). The neurobiology of non-suicidal self-injury (NSSI): A review. Suicidology Online, 3, 24-32.

Hackmann, A., Bennett-Levy, J., \& Holmes, E. A. (2011). Oxford Guide to Imagery in Cognitive Therapy. New York: Oxford University Press.

Hackmann, A., Clark, D. M., \& McManus, F. (2000). Recurrent images and early memories in social phobia. [Clinical Trial

Comparative Study

Randomized Controlled Trial

Research Support, Non-U.S. Gov't]. Behaviour research and therapy, 38(6), 601-610. 
Hackmann, A., \& Holmes, E. A. (2004). Reflecting on imagery: a clinical perspective and overview of the special issue of memory on mental imagery and memory in psychopathology. Memory, 12(4), 389-402. doi: 10.1080/09658210444000133

Harris, J. (2000). Self-harm: cutting the bad out of me. [Research Support, Non-U.S. Gov't]. Qualitative health research, 10(2), 164-173.

Himber, J. (1994). Blood rituals: self cutting in femal psychiatric patients. Psychotherapy, 31, $620-663$.

Holmes, E. A., Crane, C., Fennell, M. J., \& Williams, J. M. (2007). Imagery about suicide in depression--"Flash-forwards"? [Research Support, Non-U.S. Gov't]. J Behav Ther Exp Psychiatry, 38(4), 423-434. doi: 10.1016/j.jbtep.2007.10.004

Holmes, E. A., Geddes, J. R., Colom, F., \& Goodwin, G. M. (2008). Mental imagery as an emotional amplifier: application to bipolar disorder. [Research Support, Non-U.S. Gov't

Review]. Behaviour research and therapy, 46(12), 1251-1258. doi: 10.1016/j.brat.2008.09.005

Klonsky, E. D., \& Muehlenkamp, J. J. (2007). Self-injury: a research review for the practitioner. [Comparative Study

Review]. J Clin Psychol, 63(11), 1045-1056. doi: 10.1002/jclp.20412

Kosslyn, S. M., Ganis, G., \& Thompson, W. L. (2001). Neural foundations of imagery. [Review]. Nat Rev Neurosci, 2(9), 635-642. doi: 10.1038/35090055

Leatham, V. (2006). Bloodletting a memoir of secrets, self-harm \& survival: New Harbinger Publications.

Mahadevan, S., Hawton, K., \& Casey, D. (2010). Deliberate self-harm in Oxford University students, 1993-2005: a descriptive and case-control study. [Comparative Study 
Research Support, Non-U.S. Gov't]. Social psychiatry and psychiatric epidemiology, 45(2), 211-219. doi: 10.1007/s00127-009-0057-x

MaSH. (2012). Manchester Self-Harm (MaSH) Project, Multi-Centre Study of Self-Harm in England Retrieved 11/01/12, from http://www.medicine.manchester.ac.uk/mentalhealth/research/suicide/prevention/mas $\underline{\mathrm{h} /}$

May, J., Andrade, J., Panabokke, N., \& Kavanagh, D. (2004). Images of desire: cognitive models of craving. Memory, 12(4), 447-461. doi: 10.1080/09658210444000061

Morrison, A. P. (2004). The use of imagery in cognitive therapy for psychosis: a case example. [Case Reports

Review]. Memory, 12(4), 517-524. doi: 10.1080/09658210444000142

Nigro, G., \& Neisser, U. (1983). Point of view in personal memories. Cognitive Psychology, $15,467-482$.

Osman, S., Cooper, M., Hackmann, A., \& Veale, D. (2004). Spontaneously occurring images and early memories in people with body dysmorphic disorder. Memory, 12(4), 428436. doi: $10.1080 / 09658210444000043$

Ottaviani, R., \& Beck, A. T. (1987). Cognitive aspects of panic disorders. Journal of Anxiety Disorders, 1(1), 15-28.

Rice, H. J., \& Rubin, D. C. (2009). I can see it both ways: first- and third-person visual perspectives at retrieval. [Research Support, N.I.H., Extramural]. Conscious Cogn, 18(4), 877-890. doi: 10.1016/j.concog.2009.07.004

Smith, J. A., Flowers, P., \& Larkin, M. (2009). Interpreatative Phenomenological Analysis. London: SAGE. 
Somerville, K., \& Cooper, M. (2007). Using imagery to identify and characterise core beliefs in women with bulimia nervosa, dieting and non-dieting women. Eat Behav, 8(4), 450-456. doi: 10.1016/j.eatbeh.2006.12.004

Spandler, H. (1996). Who's hurting who? Young people, self-harm and suicide. Manchester: 42nd Street.

Speckens, A. E., Hackmann, A., Ehlers, A., \& Cuthbert, B. (2007). Imagery special issue: intrusive images and memories of earlier adverse events in patients with obsessive compulsive disorder. J Behav Ther Exp Psychiatry, 38(4), 411-422. doi: 10.1016/j.jbtep.2007.09.004

Van Moffaert, M. M. (1990). Self-mutilation: diagnosis and practical treatment. [Review]. Int J Psychiatry Med, 20(4), 373-382. 